\title{
Téoros
}

Revue de recherche en tourisme

\section{Le tourisme à Québec et dans sa grande région. Une industrie, des enjeux}

\section{Pierre Labrie et Linda Girard}

Volume 12, numéro 1, mars 1993

Québec, capitale et région touristiques

URI : https://id.erudit.org/iderudit/1078193ar

DOI : https://doi.org/10.7202/1078193ar

Aller au sommaire du numéro

Éditeur(s)

Université du Québec à Montréal

ISSN

0712-8657 (imprimé)

1923-2705 (numérique)

Découvrir la revue

Citer cet article

Labrie, P. \& Girard, L. (1993). Le tourisme à Québec et dans sa grande région.

Une industrie, des enjeux. Téoros, 12(1), 3-6. https://doi.org/10.7202/1078193ar d'utilisation que vous pouvez consulter en ligne.

https://apropos.erudit.org/fr/usagers/politique-dutilisation/ 


\title{
Le tourisme à Québec et dans sa grande région. Une industrie, des enjeux ${ }^{(1)}$
}

\author{
Pierre Labrie avec la collaboration de Linda Girard*
}

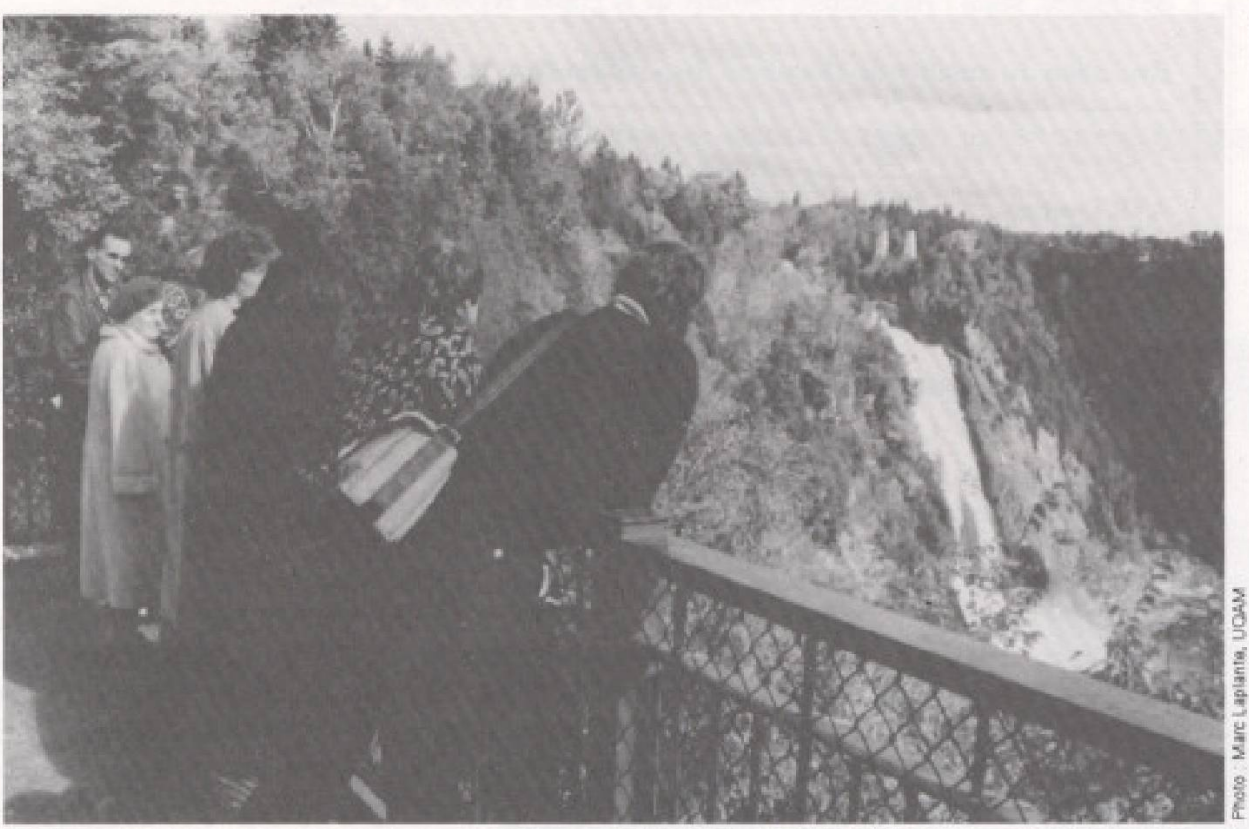

Québec et sa région pewvent pronettre une vaste gamme de plaisirs touristiques.

\section{Un peu d'histoire}

En 1826, une des premières chroniques de voyage publićes au Québec relate un voyage touristique au site de la Chute Montmorency.

Pourtant pour plusieurs, le tourisme est une industrie jeune qui a pris son envol avec l'accessibilité au transport aérien et la disponibilité de temps de vacances. Dans la région de Québec - et dans la vieille ville en particulier-le tourisme est une industrie qui a acquis ses lettres de noblesse des le $19^{*}$ siècle. En 1993, Québec célèbre le $100^{\circ}$ anniversaire de la construction du Château Frontenac.

Dans la foulée d'Expo 67, des Jeux Olympiques de 1976, de l'ouverture du Québec sur le monde, la construction des stations de ski du Mont-Sainte-Anne et de Stoneham et la construction de quatre nouveaux complexes hôteliers et du centre des congrès 1974, 1'industrie touristique de Québec s'est donné les structures que nous lui connaissons aujourd'hui.

Monsieur Pierre Labrie est directeur de l'Office du tourisme et des congres de la Communauté urbaine de Québec: Madarne Linda Girard est conseillière en planification stratégiqué et marketing a l'OTCCUO.
Au début des années 1980 , plus de 130 millions de dollars étaient investis dans les infrastructures et l'aménagement du VieuxPort, du Musée de la civilisation et des quartiers périphériques. En 1985, la ville de Québec était reconnue joyau du patrimoine mondial par l'UNESCO. En 1989, Charlevoix est reconnue réserve de la biosphère. Depuis, la ville et la grande région continuent a investir dans le développement de leurs produits sur la base des acquis développés depuis cette époque. En 1993, ces infrastructures seront complétées par l'inauguration du nouveau parc de la chute Montmorency ( 15 millions) et laconstruction d'un nouveau Centre de congrès (81 millions).

\section{Quelques faits et chiffres}

Onévalue à 4042000 le nombre de voyagespersonnes par des touristes de toutes origines géographiques dans la région métropolitaine de recensement de Québec en 1990.

Le tourisme à Québec (RMR) représente $21,7 \%$ de l'ensemble de cette industrie au Québec; le nombre de nuitées estimé s'établissent à 12159000 en 1990; les recettes brutes ont été évaluées a 875 millions \$ pour la même année.

Les emplois directs crées par les secteurs de l'hébergement et de la restauration dans la région métropolitaine de Québec sont estimés à plus de 24000 en 1992. Les emplois additionnels induits dans les secteurs du divertissement, loisirs et services sont estimes à près de 9000 . Ces emplois associés au secteur touristique (32769) représentent donc près de $10,0 \%$ de l'emploi total, ce qui est comparable au nombre d'emplois maintenu par le secteur manufacturier. Dans la région métropolitaine de Québec, le tourisme vient immédiatement après le secteur du commerce de détail, l'administration publique et celui des services médicaux en terme d'emplois crús. Il précelde les secteurs de la construction, des transports, de l'assurance et de l'enseignement.

\section{La clientèle}

En 1990, la provenance de la clientèle touristique de la région était la suivante: $48,1 \%$ venaient du Québec; $25,9 \%$, des Êtats-Unis; $18,9, \%$ de l'international (outre-mer); $7,1 \%$, du reste du Canada (autres provinces canadiennes). Soixante-quinze pour cent de la clientèle touristique de la grande région de Québec utilise l'automobile ou l'autocar comme mode de transport.

L'estimation des buts des voyages réalisésen 1990 dans la région de Québec s'établit comme suit: $37,5 \%$ pour le tourisme d'agrément; $35,5 \%$ pour les visites de parents et d'amis; $21,8 \%$ pour les affaires et congrès et $5,2 \%$ pour d'autres buts.

Enfin, notons quelques autres données significatives de l'importance du tourisme sur le territoire de l'Association touristique de la région de Québec : 900 établissements de restaurations; 31 terrains de camping; 212 établissements hồteliers; 21 golfs publics; 6 stations de ski; 6 bases de plein-air; 5 centres de villégiature; 41 musées, etc., auxquels il faut associer toutes les entreprises de services et de détail pour qui la clientèle touristique représente quand même une part signifi- 
cative de leur activite, que ce soit le commerce de détail, les stations services, etc.

\section{Principales caractéristiques du produit touristique régional}

Le Plan de développement touristique de la région de Québec, publié en 1989 , a permis de cerner les principales forces et faiblesses du produit de la région. En voici quelquesunes:

\section{Les forces}

De façon générale, il est juste de dire que notre produit régional ne souffre pas trop au chapitre des potentiels offerts par la région: une concentration d'attraits diversifiés; une culture francophone vivante manifeste lors de nombreux événements; l'exceptionnelle beauté du site de Québec, de la région et de Charlevoix; l'unicité du quartier historique, des fortifications et de la Citadelle; la présence du fleuve Saint-Laurent; un produit hivernal fort caractérisé par: une ville pittoresque, une région attrayante sous la neige, le ski alpin, le ski de fond, la motoneige et le Carnaval; desévénements majeurs récurrents: le Festival d'été et le Camaval; une grande variété d'activités récréatives et de plein air facilement accessibles et à proximité de la ville; une région qui, par son exotisme et sa situation sur le continent américain, présente un grand potentiel pour la tenue de congres: une expérience culinaire variée et de grande qualité; la qualité de vie offerte par le milieu; de bonnes liaisons routières avec les régions périphériques, Montréal et les grandes villes du Nord-Est américain; une présence gouvernementale, Québec Capitale.

Qui dit forces dit Également reconnaitre l'existence de certaines faiblesses et contraintes. Soulignons, entre autres: Québec et sa région sont encore aujourd'hui un des secrets les mieut gardés de l'industrie touristique canadienne et internationale; un manque général d'organisation des produits touristiques, de façon à mieux les faire connaiture et à les rendre plus accessibles et plus facilement commercialisables; le peu d'intégration des produits culturels à la promotion de la région; la déficience des équipements et infrastructures pour la tenue de congrès d'envergure (tout au moins jusqu'en 1996); la sous-exploitation du potentiel d'attrait de Québec comme Capitale; le manque d'accessibilité aux plans d'eauet une carence destations touristiques et de villégiature dans la région immédiate de Québec: le manque de liaisons aériennes adéquates notamment

Évolution du taux d'occupation en \% dans la région touristique de Québec

\begin{tabular}{llllllll}
\hline & 1985 & 1986 & 1987 & 1988 & 1989 & 1990 & 1991 \\
\cline { 2 - 7 } ATR Québec & 58,4 & 60,3 & 60,9 & 56,5 & 56,4 & 53,5 & 52,1 \\
\hline
\end{tabular}

Les résultats de 1992 démontrent une baisse d'environ $7 \%$ du taux d'occupation hôtelière del'ATR de Québec, selon l'Association hôtelière de la grande région de Québec.

Évolution du nombre de chambres disponibles dans la région touristique de Québec

\begin{tabular}{lrrrrrrr}
\hline & 1985 & 1986 & 1987 & 1988 & 1989 & 1990 & 1991 \\
\cline { 2 - 7 } ATR Québec & 7943 & 8055 & 8723 & 9373 & 10184 & 10496 & 10169 \\
\hline
\end{tabular}

On remarque un accroissement de $28 \%$ du nombre de chambres disponibles depuis 1985 . En 1991. I'ATR de Québee disposait d'un nombre total de 10169 chambres; ce nombre est resté stable en 1992.

pour les produits: affaires-congrès, courts séjours d'agrément et ski alpin; la faible notoriété de la région sur les principaux marchés-cibles; le développement inégal de la culture touristique.

Dans uncontexteévolutif, larégiondeQuébec poumait profiter, à court et moyen termes, de certaines opportunités de développement.

- Leproduit touristique régional possede les caractéristiques qui seront recherchées par les consommateurs durant les prochaines années: la qualité, l'originalité, la différence et l'inédit.

- Le fractionnement des vacances d'agrément et, par conscquent, la multiplication des séjours de ce type pourront nous avantager.

- La croissance de la demande pour les produits circuits courts ainsi que pour les centres de villégiature de Québec et de Charlevoix.

- L'intérêtcroissant des touristes d'outremer - surtout les Européens francophones - pour la grande région de Québec en tant qu'etape sur un circuit nordaméricain; prolongation de la durée de séjour dans la région.

- L'implantation prochaine d'infrastructures adaptées et efficaces pour la tenue de congrès, de foires et d'expositions. commerciales.

- Le développement anticipé de croisières dans l'axe Nouvelle-AngleterreQuébec-Mont.

- La hausse de la demande pour des circuits périurbains de la part des touristes. d'affaires et de congrès.

- La hausse de la demande pour les produits de plein air et d'aventure douce a proximité d'une grande ville.
Des balises pour mieux planifier I'avenir

Au cours des années 1980, portée par une économie en croissance et une industrie touristique en plein essor, la grande région touristique de Québec en a profité pour se doter d'un Plan de développement touristique. Ce travail, réalisé de 1987 a 1989, a constitué un tournant majeur pour les intervenants du milieu. En effet, plus de 200 entreprises et regroupements issus du milieu des affaires, du monde municipal et des associations ont travaillé conjointement à l'élaboration d'un cadre de développement du tourisme. Pour la première fois, il y eu concertation réelle entre les intervenants des MRC de Portneuf, Jacques-Cartier, Côte-de-Beaupré et Ile d'Orleans et le territoire de la Communauté urbaine de Québec. Cette démarche de concertation a également engendré divers projets susceptibles de contribuer à moyen et long termes a l'essor de l'industrie touristique régionale.

\section{Rôle de l'OTCcua}

Dans un contexte de plus en plus compétitif et face à une concurrence accrue, il importe pour tous les intervenants de partager une compréhension commune desenjeux del'industrie touristique dans la région de Québec, de la place qu'elle occupe dorénavant dans l'économie régionale et de la contribution qu'elle peut apporter ả court et à long termes. L'Office du tourisme et des congrès de la Communauté urbaine de Québec existe dans son cadre actuel depuis 1984. Service à part entière de la CUQ, I'Office regroupe plus de 475 entreprises et est reconnu comme Association touristique régionale (ATR) par le 
ministère du Tourisme duQuébec. Il dispose pour l'exercice 1993 d'un budget de 5,1 millions de dollars dont 3,3 proviennent des contributions municipales; le solde provient des programmes a frais de participations gouvemementales partagés, et des contributions du secteur privé. La mission de l'OTCCUQ, actualisceen 1992 dans le cadre d'une démarche de planification stratégique, peut être résuméce ainsi:

*En tant qu'Association touristique régionale de Québecetassumant les rôles d'organismede concertation et deporteparole de l'industrie, l'Office doit contribuer activement à la prospérité 6 conomique de l'industrie touristique en favorisant, de façon coordonnée et integrée, son marketing, son développement, l'accueil des touristes et l'information du milieu.s

Grâce au travail réalisé au cours des demières années par les intervenants régionaux, l'industrie touristique régionale dispose dorénavant de plusieurs outils pour orienter ses démarches, soit: le Plan de développement touristique de 1989; la Planification stratégique de l'OTC; le Plan marketing de l'OTC; et, depuis avril 1992, une entente interrégionale qui a permis de consolider les relations entre la CUQ et ses partenaires des MRC de Portneuf, Jacques-Cartier, Ile d'Orléans, Côte-de-Beaupré.

Fort de cette nouvelle concertation, 1 'industrie touristique régionale est à même de définir maintenant les limites réalistes du travail qu'elle peut et doit réaliser au cours des cinq prochaines années.

\section{Principaux enjeux}

L'industrie doit s'inspirer des orientations, des objectifs et des conclusions du Plan de développement, des interventions identifiées par ses partenaires, des orientations communiquées par le ministère du Tourisme et des résultats observés sur le terrain, tant sur le territoire de la ville de Québec et de la Communauté urbaine, que sur celui des municipalités régionales de comté.

Dans un contexte économique plus difficile, il faudra de plus éviter de créter chez les differents partenaires du milieu des attentes démesurées qui risqueraient d'outrepasser la capacité même de l'industric à réaliser un plan d'action.

\section{Les objectifs de développement de l'industrie}

D'un point de vue strictementéconomique, il va de soi que l'objectif des actions régionales en matière de tourisme sera l'accroissement des recettes et, par conséquent, des retombées régionales générées. C'est d'ailleurs là le premier objectif de l'Énoncé de politique et du Plan d'action du ministère du Tourisme du Québec. Du point de vue plus spécifique de l'industrie touristique régionale, il faut en priorite: sFavoriser l'accroissement de la demande jusqu'à un niveau capable de susciter la confiance des investisseurs:.

Pour y arriver, l'industrie devra satisfaire les sous-objectifs suivants:

- Accroissement de la durée de séjour: il s'agit de prolonger la durée de séjour moyenne des visiteurs dans la région qui a diminue d'environ $25 \%$ au cours des cinq dernières années, pour retrouver un niveau qui pourrait contribuer à accroitre les recettes touristiques.

- Étalement de la demande saisonnière dans le temps comme dans l'espace: développement d'activités artistiques et culturelles à l'automne et au printemps. Trouver le moyen de mobiliser les intervenants culturels pour relever ce défi. Mise en valeur des produits touristiques disponibles sur les territoires périphériques au centre-ville de Québec.

- Coordination et optimisation des efforts promotionnels: mises en commun des ressources coordonnés par l'Office du tourisme et des congrès et impliquant le partenariat privé, la publicité coopérative et les promotions conjointes afin de capitaliser sur l'ensemble des ressources de la région.

- Intensification de la concertation par l'entremised'un partenariat renouvelé: développement des relations interrégionales, notamment avec l'Association touristique de Charlevoix et celle de Beauce-Appalaches, afin de mettre en commun des données et des ressources et pour viser à prolonger la durée de sejour et entreprendre des activités de commercialisation intégrées.

- Accroissement de la compétitivité de l'industrie touristique régionale: mise en place des systèmes d'information, de recherches-marketing et de connaissance de la concurrence afin de mieux positionner la région de Québec et capitaliser davantage sur nos forces.
- Developpement d'une culture touristique dans la population: sensibiliser la population locale à l'importance d'accueillir favorablement le touriste, la former adéquatement et lui faire jouer un rôle de promoteur auprès de clientèles susceptibles de nous fréquenter.

- Consolidation et renowvellement du produit touristique régional: améliorer la capacité d'attraction du produit touristique par la mise en valeur des attraits de la région, consolider les produits touristiques existants en tenant compte des besoins des clientelesetdes nouvelles tendances qui s'expriment, assurer le développement de manière harmonieuse, respectueusedel ${ }^{4}$ environnement et soucieuse de la qualite de vie des citoyens.

- Atteinte de l'excellence touristique: développer chez lesentreprises touristiques de la région un réflexe, une préoccupation constante pour laqualité totale en matière de mise en marché, de développement de l' offre et de formation de la main-d'oeuvre.

\section{Les axes stratégiques et les actions prioritaires}

Afinderendrecepland'action véritablement opérationnel, les objectifs de développement de l'industrie touristique devront être traduits en axes d'intervention et en actions prioritaires, lesquels sont présentés ci-après.

ler axe: l'organisation et la coordination du développement touristique

La mise en oeuvre du plan d'action requiert, en premier lieu: d'assurer la concertation des intéressés à tous les niveaux de l'industrie; d'assurer une convergence des actions; de renouveler le partenariat;

pour une organisation et une coordination plus efficaces des activités de développement et de promotion.

2e axe: la mise en marché du produit régional

Les objectifs du plan d'action nécessitent, pour être réalisés, des efforts particuliers en matière de mise en marché du produit régional. Les actions prioritaires sont les suivantes: préparer et mettre en oeuvre une planification marketing des 1993; orienter les ressources et les capacités régionales vers la recherche, le développement, la planification et l'évaluation des stratégies et les plans 
d'actions marketing pour les marchés du tourisme d'agrément et d'affaires; favoriser la mise sur pied de forfaits et assurer leur promotion; mieux cibler des clientèles; àcourt terme, accroitre les efforts de promotion du tourisme d'agrément; meture en place un processus $d^{+}$evaluation des perfomances touristiques etdel'impact des stratégies mises en ceuvre afin de pouvoir réagir rapidement à l'évolution de l'environnement touristique.

3e axe: la consolidation et le renouvellement de l'offre touristique

Bien qu'à court terme, notre priorité d'action porte davantage sur la mise en marché, l'industrie touristique régionale ne doit pas négliger pour autant la consolidation et le renouvellement de l'offre comme facteur de soutien de sa position concurrentielle sur les marchés. En parlant de développement de l'offre, nous ne référons pas uniquement aux immobilisations mais aussi à la mise en oeuvre de produits nouveaux utilisant pleinement les possibilités que présentent les ressources du milieu et l'offre touristique régionale. Diverses actions doivent être priorisées, à savoir: développer des circuits intrarégionaux ou périurbains en collaboration avec les Offices de zone de Portneuf, de la Jacques-Cartier, de la Côte-de-Beaupré et de l'Ile d'Orleans; développer des circuits interrégionaux en collaboration avec les Associations touristiques régionales de Charlevoix, de Chaudière-Appalaches et du Coeur-du-Québec; intégrer nos produits régionaux au positionnement culture-nature préconisé par le ministère du Tourisme (Québec, joyau du patrimoine mondial); favoriser la mise en valeur des attraits et des berges du fleuve Saint-Laurent (parcs, plages, pistes cyclables) sur l'ensemble du territoire; contribuer au developpement de l'image et du produit Québec Capitale; promouvoir l'amélioration du réseau routier, de la signalisationet du réseau d'accueil touristique; travailleràaméliorerl'accessaćrien à la région: en favorisant la mise en place de liaisons acriennes directes avec un plus grand nombre de villes de l'Est de l' Amérique du Nord; en encourageant l'organisation de forfaits (ski, culture, escapades de fins de semaine) par les grossistes et les intervenants touristiques régionaux; en favorisant les liaisons directes entre Montréal, Toronto et Québecà l'occasion des congrès et de foires $\mathrm{d}^{*}$ envergure; consolider et assurer la réalisation annuelle ou bi-annuelle des événements majeurs qui contribuent à créer notre image de marque tout en favorisant l'étalement saisonnier: Carnaval de Québec;, Festival d'été;
Médiévales; Transat; Coupes du monde; soutenir des projets moteurs - tel Québec 2002 - qui contribuent à bonifier le potentiel de développement de la région; soutenir les gens d'affaires et les appuyer dans leurs efforts pour solliciter des congrès intemationaux.

\section{Conclusion}

Le tourisme: une industrie importante à faire connaître et reconnaître

Le tourisme à Québec comme dans la grande région est très important. Bien qu'installée aux premiers rangs de l'activité économique régionale, l'industrie touristique surpasse en réalité des secteurs souvent considérés comme ayant un rôle plus significatif et plus important. Les intervenants touristiques doivent encore aujourd'hui convaincre les autres acteurs du développement économique régional de la pertinence d'investir temps, argent et ressources humaines dans le domaine. De l'avis de plusieurs experts qui se sont exprimés sur le sujet, le tourisme dans la région represente fort probablement le moteur de développement économique le plus susceptible de contribuer à créer rapidement et à un prix raisonnable des emplois permanents qui favorisent la reprise économique régionale au cours des cinq prochaines années.

\section{Le développement touristique :} un rôle pour tous

L'industrie touristique ne doit se developper ni par défaut ni par inertie; elle doit le faire par volonté. La ville et la région de Québec offrent un produit unique non seulement au Québec mais aussi au Canada et en Amérique du Nord. C'est un produit dans lequel nous devons collectivement continuer à investir, tant au plan du développement des avantages concurrentiels dont nous disposons qu'au plan de la mise en marché nécessaire pour assurer le développement de notre industrie.

Rassemblés autour des mêmes objectifs, les partenaires de l'industrie devront unifier leurs gestes, s'assurer de la complémentarité de leurs efforts et ainsi créer une synergie qui soit une veritable garantie d'avenir pour l'industrie. L'industrie touristique reconnaît comme principe que les secteurs public et privé ne peuvent et ne doivent agir de façon isolée et indépendante. II faut donc solliciter les capacites et les compétences des divers intervenants de manière à mettre en place, dans la région, les conditions favorables à l'essor du tourisme. Dans cette perspective, les rôles des differents partenaires sont donc les suivants: l'entreprise privée doit de plus en plus être le moteur du développement. Elle doit assumer la prise en charge des services touristiques de base, les activités et les attraits, investir pour renouveler l'offre et collaborer étroitement à la mise en marché du produit régional; les intervenants touristiques, economiques et politiques régionaux doivent mettre en commun ressources financières, humaines et vision d'avenir afin de capitaliser sur les acquis et les opportunités collectives; les municipalités, les municipalités régionales de comté et la Communauté urbaine de Qućbec doivent contribuer au maintien d"un environnement de qualité et propice à l'essor touristique par l'entremise de leurs interventions enmatièred'aménagement du territoire et d'ubanisme et de réglementation; les gouvemements supérieurs, au provincial comme au fédéral, doivent mettre à la disposition de la région les ressources financières et prendre les décisions requises pour que le produit touristique évolue en amont des tendances de l'industrie et puisse augmenter sa part de marché au fil des années; enfin, l'OTCCUQ doit assumer et affirmer son leadership dans les domaines de la concertation régionale, de la mise en marché de l'accueil et de la planification du développement de l'industrie touristique.

L'industrie touristique doit également s' impliquer dans le domaine de la formation d'une main-d'ceuvre qualifiée à tous les niveaux, en collaboration étroite avec les établissements d'enseignement, les entreprises et les ministères concernés, par la mise sur pied de programmes d'études, de programmes de formation sur mesure, de campagnes de valorisation des métiers et des professions reliés au tourisme et de colloques de formation.

Les intervenants touristiques régionaux doivent par ailleurs développer un modele de gestion du développement de l'industrie qui instaure un partage des rôles, des responsabilités, des actionset des interventions selon les capacités et les compétences de chacun.

Une attitude à préconiser pour l'avenir La concurrence de plus en plus vive impose au secteur touristique un redéploiement en force de manière à devenir plus compétitif. Notre objectif peut se traduire par ces simples mots: qualité - excellence - performance. -

\section{NOTE}

(1) Le texte qui suit a été adapté d'une presentation taite par monsieur Labrie le 14 novembre 1992 dans le cadre de l'assemblé géndrale annuelle du Conseil régional de concertation et de diveloppement de la région de Québec. 